\title{
Short Door-to-Needle Times in Acute Ischemic Stroke and Prospective Identification of Its Delaying Factors
}

\author{
Sander M. Van Schaik ${ }^{a}$ Saskia Scott ${ }^{b}$ Lonneke M.L. de Lau ${ }^{b}$ \\ Renske M. Van den Berg-Vos ${ }^{a}$ Nyika D. Kruyt ${ }^{\mathrm{C}}$

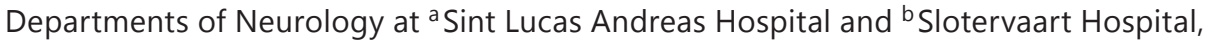 \\ Amsterdam, and ${ }^{\mathrm{C}}$ Department of Neurology, Leiden University Medical Center, \\ Leiden, The Netherlands
}

\section{Key Words}

Acute ischemic stroke - Acute management of stroke - Acute stroke thrombolysis .

Emergency neurology · Quality improvement · Thrombolytic therapy

\begin{abstract}
Background: The clinical benefit of intravenous thrombolysis (IVT) in acute ischemic stroke is time dependent. Several studies report a short median door-to-needle time (DNT; $20 \mathrm{~min}$ ), mainly in large tertiary referral hospitals equipped with a level 1 emergency department, a dedicated stroke team available $24 / 7$, and on-site neuroimaging facilities. Meanwhile, in daily practice, the majority of stroke patients are admitted to secondary care hospitals, and in practice, even the generous benchmark of the American Heart Association (a DNT of 60 min in $>80 \%$ of the cases) is met for a minority of patients treated with IVT. The first objective of our study was to investigate if, in a secondary care teaching hospital rather than a tertiary referral hospital, similar short DNTs can be accomplished with an optimized IVT protocol. Our second objective was to prospectively identify factors that delay the DNT in this setting. Methods: A multicenter, consecutive cohort study of patients treated with IVT in one of two secondary care teaching hospitals. In both hospitals, data of consecutive stroke patients as well as median DNTs and factors delaying this were prospectively assessed for each patient. Multivariable logistic regression analysis was used to evaluate associations between patient-related and logistic factors with a delayed (i.e. exceeding $30 \mathrm{~min}$ ) DNT. Results: In total, 1,756 patients were admitted for ischemic stroke during the study period. Out of these, 334 (19.0\%) patients were treated with IVT. The median DNT was 25 min (interquartile range: $20-35$ ). A total of $71 \%$
\end{abstract}

S.M.V.S. and S.S. contributed equally to this work.

Sander M. Van Schaik, MD

Department of Neurology, Sint Lucas Andreas Ziekenhuis

Jan Tooropstraat 164

NL-1061 AE Amsterdam (The Netherlands)

E-Mail svschaik@gmail.com 
Van Schaik et al.: Short Door-to-Needle Times in Acute Ischemic Stroke and Prospective Identification of Its Delaying Factors

$(n=238)$ had a DNT below $30 \mathrm{~min}$. In $63 \%$ of the patients treated with IVT the DNT was delayed by at least one factor. Patients without any delaying factor had a 10 min shorter median DNT compared to patients with at least one delaying factor $(p<0.001)$. The following factors were independently associated with a delayed DNT: uncertainty about symptom onset, uncontrolled blood pressure, fluctuating neurological deficit, other treatment before IVT, uncertainty about (anti-)coagulation status, other patient-related factors, and incorrect triage. Conclusions: Short median DNTs can also be accomplished in secondary care. Despite the short DNTs, several delaying factors were identified that could direct future improvement measures. This study supports the view that as a performance measure, the current DNT targets are no longer ambitious enough and it adds to the knowledge of factors delaying the DNT.

(c) 2015 S. Karger AG, Basel

\section{Introduction}

Intravenous thrombolysis (IVT) significantly improves clinical outcome in acute ischemic stroke, but the clinical benefit of this treatment rapidly declines with the passing of time [1]. Still, the time from hospital admission to treatment, the so-called door-to-needle time (DNT), is often delayed for avoidable reasons. Several studies report a short median DNT mainly in large tertiary referral hospitals equipped with a level 1 emergency department, a dedicated stroke team available 24/7, and on-site neuroimaging facilities [2-4]. Meanwhile, in daily practice, the majority of stroke patients are admitted to smaller secondary care hospitals [5, 6], and in practice, even the generous benchmark of the American Heart Association (a DNT of $60 \mathrm{~min}$ in $>80 \%$ of the cases) $[7,8]$ is met for a minority of patients treated with recombinant tissue plasminogen activators (rt-PA) $[9,10]$. Moreover, even when an optimized IVT protocol is operational, the DNT is potentially delayed by various patient-related and logistic factors. The identification of these factors could facilitate further shortening of the DNT [11], but so far, no prospective documentation of such factors has been published. The first objective of our study was therefore to investigate if, in a secondary care rather than a tertiary referral hospital, similarly short DNTs can be accomplished with an optimized IVT protocol. Our second objective was to prospectively identify factors that delay the DNT in this setting.

\section{Methods}

Data from two secondary care teaching hospitals in the Netherlands, the Slotervaart hospital (SH) and the Sint Lucas Andreas Hospital (SLAH), were collected. Both hospitals are situated on the western outskirts of Amsterdam within a 2.5-mile distance and offer roundthe-clock rt-PA treatment. The stroke team in both hospitals consists of an on-call neurologist, an on-site neurology resident available 24/7, a stroke and emergency department nurse, a radiology technician, and a laboratory analyst. In both hospitals, the neurology residents witness several rt-PA treatments and/or receive focused training before they run a shift on their own. Logistics are largely similar in both hospitals. The emergency department receives a prenotification from the ambulance announcing a patient potentially eligible for rt-PA treatment (i.e., a patient with an acute focal neurological deficit of $<4.5 \mathrm{~h}$ duration), allowing for preparation of the acute stroke team and clearing of the CT scan. Glucose levels and the international normalized ratio (INR) are checked with a point-of-care device, intravenous access is obtained if not already available, and blood is drawn, but rt-PA treatment is not delayed for (platelet count) results, unless there is clinical suspicion or a history of severe thrombocytopenia. At the start of the study period, all patients were asked for their weight. 


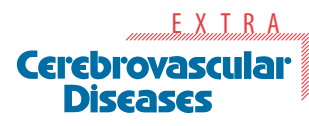

\begin{tabular}{l|l}
\hline \multicolumn{2}{l}{ Cerebrovasc Dis Extra 2015;5:75-83 } \\
\hline DOI: 10.1159/000432405 & $\begin{array}{l}\text { C 2015 S. Karger AG, Basel } \\
\text { www.karger.com/cee }\end{array}$ \\
\hline
\end{tabular}

Van Schaik et al.: Short Door-to-Needle Times in Acute Ischemic Stroke and

Prospective Identification of Its Delaying Factors

Table 1. Potential patient-related and logistic factors delaying the DNT

\begin{tabular}{ll}
\hline Patient-related factors & Logistic factors \\
\hline - Uncertainty about symptom onset & - Incorrect triage \\
- Unknown medical history & - Insufficient personnel \\
- Uncontrolled blood pressure & - Difficulties with drip or urinary catheter insertion \\
- Fluctuating neurological deficit & - Difficulties with weighing the patient \\
- Patient has to undergo other treatment before IVT & - CT scan occupied \\
- Uncertainty about (anti-)coagulation status & - Technical problems \\
& - Laboratory results delayed \\
& - No medication available \\
& - Waiting for consent from the patient or family \\
& - Patient transferred from another institution \\
\hline
\end{tabular}

Original from [11].

If asking was not possible or when the weight was unknown, it was either estimated or determined with a scale. Since 2013, both hospitals determine body weight by using a bed with a built-in scale. In both hospitals, patients have to be transported by elevator to the CT suite on the first floor. The decision to treat with rt-PA is based on a non-contrast head CT, and the rt-PA bolus is administered while the patient is still on the CT table, directly followed by a continuous infusion. Since IVT with rt-PA is the standard of care for the treatment of acute ischemic stroke, the DNT was not delayed by obtaining informed consent. Neither hospital actively lowers uncontrolled hypertension (>185/110 mm Hg). Instead, blood pressure is monitored every $10 \mathrm{~min}$, and in case of a spontaneous drop within the time window for IVT, rt-PA is administered.

In both hospitals, data of consecutive stroke patients are prospectively collected and include demographic variables and the medical history. In addition, for patients treated with rt-PA, the following parameters are documented: the symptom-to-needle time (SNT), symptom-to-door time (SDT), and the DNT. The DNT is defined as the time between the moment the patient first enters the door of the facility (and for patients already hospitalized, the moment of first consultation of a neurologist) and administration of the intravenous bolus with rt-PA. Both hospitals prospectively document previously published factors that potentially delay the DNT (table 1) [11]. Because increasingly short DNTs could potentially lead to a less accurate diagnosis and to more rt-PA-related complications, we prospectively documented the diagnosis on discharge to retrieve stroke mimics and possible hemorrhagic complications related to rt-PA treatment. For intracerebral hemorrhage, we used a pragmatic definition of blood on a CT scan explaining any clinical relevant neurological deterioration during hospital admission. The SH database also includes stroke severity on admission as measured with the National Institutes of Health Stroke Scale (NIHSS) score, the clinical location of the stroke, and the vascular risk factors. These data were reconstructed from medical records for patients in the SLAH (no missing values). Patients were included between October 2011 and October 2013. We obtained institution review board approval in both hospitals to conduct this analysis.

\section{Statistics}

Dichotomous data are described as numbers and percentages, and continuous data are presented as means with standard deviations $( \pm \mathrm{SD})$. For non-normally distributed data, median values and interquartile ranges (IQR) are presented. Differences between the hospi- 

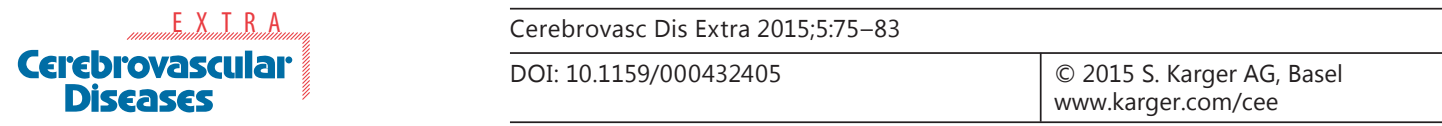

Van Schaik et al.: Short Door-to-Needle Times in Acute Ischemic Stroke and

Prospective Identification of Its Delaying Factors

Table 2. Patient characteristics per hospital

\begin{tabular}{lcccc}
\hline Variable & $\begin{array}{l}\text { Total } \\
(\mathrm{n}=334)\end{array}$ & $\begin{array}{l}\text { SH } \\
(\mathrm{n}=63)\end{array}$ & $\begin{array}{l}\text { SLAH } \\
(\mathrm{n}=271)\end{array}$ & $\mathrm{p} \mathrm{value}^{\mathrm{a}}$ \\
\hline Mean age \pm SD, years & $72 \pm 13.0$ & $74 \pm 12.0$ & $72 \pm 13.2$ & 0.196 \\
Males & $176(53)$ & $28(44)$ & $148(55)$ & 0.162 \\
History of vascular risk factors & & & & \\
$\quad$ (p)AF/valvular disease & $43(12.9)$ & $10(15.9)$ & $33(12.2)$ & 0.410 \\
Hypertension & $192(58)$ & $35(56)$ & $157(58)$ & 0.778 \\
Myocardial infarction/coronary disease & $102(31)$ & $19(30)$ & $83(31)$ & 1.00 \\
$\quad$ Dyslipidemia & $124(37)$ & $24(38)$ & $100(37)$ & 0.885 \\
$\quad$ Diabetes mellitus & $62(18.6)$ & $11(17.5)$ & $51(18.8)$ & 1.00 \\
$\quad$ Previous TIA/ischemic stroke & $100(30)$ & $25(40)$ & $75(28)$ & 0.068 \\
$\quad$ Smoking & $109(33)$ & $15(24)$ & $94(35)$ & 0.103 \\
Clinical stroke localization & $76(23)$ & $17(27)$ & $59(22)$ & 0.405 \\
$\quad$ Right cortical & $146(44)$ & $26(41)$ & $120(44)$ & 0.676 \\
Left cortical & $34(10.2)$ & $10(15.9)$ & $24(8.9)$ & 0.107 \\
$\quad$ Right subcortical & $20(6.0)$ & $0(0)$ & $20(7.4)$ & 0.019 \\
Left subcortical & $7(2.1)$ & $0(0)$ & $7(2.6)$ & 0.355 \\
Occipital & $42(12.6)$ & $8(12.7)$ & $34(12.5)$ & 1.00 \\
Posterior circulation & $6(3-10)$ & $6(3-13)$ & $6(4-10)$ & 0.556 \\
Median admission NIHSS score (IQR) & & & & \\
\hline
\end{tabular}

Values are $\mathrm{n}(\%)$, unless otherwise indicated.

(p) $\mathrm{AF}=($ Paroxysmal $)$ atrial fibrillation; TIA = transient ischemic attack.

${ }^{a}$ Determined by use of the independent sample $t$ test for age, the Mann-Whitney U test for the NIHSS score on admission, and the $\chi^{2}$ test for categorical variables.

tals and patient groups were analyzed by the Mann-Whitney $U$ test or $t$ test for continuous parameters and the $\chi^{2}$ test for categorical parameters. A two-tailed $p$ value $<0.05$ indicated statistical significance.

A delayed DNT was defined as a DNT $>30 \mathrm{~min}$. This cut-off was used because it is the goal set by the Dutch Stroke Knowledge Network. First, univariable analysis was used to assess the crude association between patient characteristics or factors potentially delaying the DNT and a delayed DNT. Second, variables with a p value $<0.10$ in the univariable analysis were entered into a multivariable logistic regression model to identify independent predictors of a delayed DNT. Because of a significant difference in the median DNT between the hospitals, we also added the treatment location to the multivariable model. All statistical analyses were carried out using IBM SPSS Statistics version 20. Statistical significance was set at $\mathrm{p}<0.05$.

\section{Results}

\section{Patients}

In total, 1,756 patients were admitted for ischemic stroke during the study period (data not shown). Out of these, $334(19.0 \%)$ patients [176 men (53\%)] were treated with rt-PA (table 2). The mean age was 72 years $( \pm 13.0)$. Cardiovascular risk factors were common: hypertension (58\%); myocardial infarction or coronary disease (31\%); prior transient ischemic attack or ischemic stroke (30\%); dyslipidemia (37\%), and current smoking (33\%). The median NIHSS score on admission was 6 (IQR: 3-10). The most common clinical stroke 


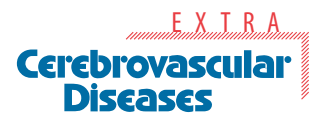

\begin{tabular}{l|l}
\hline \multicolumn{2}{l|}{ Cerebrovasc Dis Extra 2015;5:75-83 } \\
\hline DOI: $10.1159 / 000432405$ & $\begin{array}{l}\text { C) 2015 S. Karger AG, Basel } \\
\text { www.karger.com/cee }\end{array}$ \\
\hline
\end{tabular}

Van Schaik et al.: Short Door-to-Needle Times in Acute Ischemic Stroke and Prospective Identification of Its Delaying Factors

Table 3. DNT, SNT, mimics, and hemorrhagic complications per hospital

\begin{tabular}{|c|c|c|c|c|}
\hline Variable & $\begin{array}{l}\text { Total } \\
(n=334)\end{array}$ & $\begin{array}{l}\mathrm{SH} \\
(n=63)\end{array}$ & $\begin{array}{l}\text { SLAH } \\
(n=271)\end{array}$ & $\begin{array}{l}\mathrm{p} \\
\text { value }^{\mathrm{a}}\end{array}$ \\
\hline Median DNT (IQR), min & $25(20-35)$ & $30(23-45)$ & $23(19-30)$ & $<0.001$ \\
\hline DNT $<30 \mathrm{~min}$ & $238(71)$ & $33(52)$ & $202(75)$ & 0.001 \\
\hline Median SNT (IQR), min & $105(79-151)$ & $115(85-155)$ & $103(75-150)$ & 0.167 \\
\hline Patients with $\geq 1$ factor delaying rt-PA treatment & $211(63)$ & $48(76)$ & $163(60)$ & 0.020 \\
\hline Median DNT (IQR) without factors delaying rt-PA treatment, min & $20(17-23)$ & $17(15-22)$ & $20(17-23)$ & 0.164 \\
\hline Median DNT (IQR) with $\geq 1$ factor delaying rt-PA treatment, min & $30(23-45)$ & $38(30-55)$ & $28(21-40)$ & $<0.001$ \\
\hline Hemorrhagic complication rate & $11(3.3)$ & $1(1.6)$ & $10(3.7)$ & 0.70 \\
\hline Symptomatic ICH $<36 \mathrm{~h}$ & $10(3.0)$ & $1(1.6)$ & $9(3.3)$ & 1.00 \\
\hline Symptomatic systemic hemorrhage $<36 \mathrm{~h}$ & $1(0.3)$ & 0 & $1(0.4)$ & 0.69 \\
\hline Stroke mimic & $9(2.7)$ & $2(3.2)$ & $7(2.6)$ & 0.680 \\
\hline
\end{tabular}

Values are n (\%), unless otherwise indicated.

${ }^{a}$ Determined by use of the Mann-Whitney U test for continuous variables and the $\chi^{2}$ test for categorical variables.

locations were left cortical (44\%) and right cortical (23\%). Except for a higher rate of the left subcortical clinical stroke location in the SLAH, the case mix of patients was similar in both hospitals (table 2).

\section{DNTs, SNTs, Stroke Mimics, and Complications}

Overall, the median DNT was 25 min (IQR: 20-35). In the SLAH, the median DNT was significantly shorter than in the SH $(23$ vs. 30 min; $p<0.001)$. Seventy-one per cent of the patients $(\mathrm{n}=238)$ had a DNT $\leq 30 \mathrm{~min}$. Patients without any delaying factor had a $10 \mathrm{~min}$ shorter median DNT compared to patients with at least one delaying factor $(p<0.001)$. The median SNT was similar between the hospitals. Symptomatic intracerebral hemorrhage (ICH) occurred in 10 patients (3.0\%). One patient $(0.3 \%)$ died from a systemic hemorrhage. During follow-up, 9 patients (2.7\%) turned out to have a stroke mimic (table 3 ).

\section{Factors Associated with a Delayed DNT}

There were no significant differences between the group with a DNT $\leq 30 \mathrm{~min}$ and the delayed DNT group for age, sex, SNT or stroke mimic rate. Patients in the group with a DNT $\leq 30 \mathrm{~min}$ had slightly more severe strokes (median NIHSS score on admission 6 vs. $5, \mathrm{p}=$ 0.054 ; table 4).

In $63 \%$ of the 334 rt-PA-treated patients, the DNT was delayed by at least one factor. In the delayed DNT group, $94 \%$ had at least one factor delaying the DNT versus $51 \%$ in the group with a DNT $\leq 30 \mathrm{~min}(\mathrm{p}<0.001)$. The most frequently reported patient-related delaying factors were fluctuating neurological deficit $(11.4 \%)$ and uncontrolled blood pressure (>185/110 mm Hg, 10.8\%; table 4). As for logistic delaying factors, the most commonly reported variables were incorrect triage $(9.3 \%)$ and technical problems $(9.0 \%$; e.a., nonfunctioning point-of-care INR-device, problems with the elevator or computer; table 4). In the $31(9.3 \%)$ patients in whom incorrect triage was registered as a factor that delayed the DNT, there was no prenotification in 10 cases, 17 patients were triaged incorrectly by the emergency department nurse, and 2 patients by the neurology resident. For the two remaining patients, the reason for the incorrect triage was not registered in the prospective database. 
Van Schaik et al.: Short Door-to-Needle Times in Acute Ischemic Stroke and

Prospective Identification of Its Delaying Factors

Table 4. Patient characteristics and factors delaying intravenous thrombolysis

\begin{tabular}{|c|c|c|c|c|}
\hline Variable & $\begin{array}{l}\text { Total } \\
(n=334)\end{array}$ & $\begin{array}{l}\mathrm{DNT} \leq 30 \mathrm{~min} \\
(\mathrm{n}=238)\end{array}$ & $\begin{array}{l}\text { DNT }>30 \text { min } \\
(n=96)\end{array}$ & $\begin{array}{l}\mathrm{p} \\
\text { value }^{c}\end{array}$ \\
\hline Mean age $\pm S D$, years & $72 \pm 13.0$ & $72 \pm 12.7$ & $73 \pm 13.8$ & 0.334 \\
\hline Males & $176(53)$ & $127(53)$ & $49(51)$ & 0.718 \\
\hline Median NIHSS score (IQR) on admission & $6(3-10)$ & $6(4-11)$ & $5(3-8)$ & 0.054 \\
\hline Median SDT (IQR) & $73(50-112)$ & $75(50-119)$ & $70(45-90)$ & 0.152 \\
\hline Stroke mimics & $9(2.7)$ & $6(2.5)$ & $3(3.1)$ & 0.720 \\
\hline$\geq 1$ factor delaying rt-PA treatment & $211(63)$ & $121(51)$ & $90(94)$ & 0.001 \\
\hline \multicolumn{5}{|l|}{ Patient-related factors } \\
\hline Fluctuating neurological deficit & $38(11.4)$ & $13(5.5)$ & $25(26)$ & $<0.001$ \\
\hline Uncontrolled hypertension ${ }^{\mathrm{b}}$ & $36(10.8)$ & $14(5.9)$ & $22(23)$ & $<0.001$ \\
\hline Other patient-related factors ${ }^{c}$ & $29(8.7)$ & $15(6.3)$ & $14(14.6)$ & 0.019 \\
\hline Uncertainty about symptom onset & $19(5.7)$ & $7(2.9)$ & $12(12.5)$ & 0.001 \\
\hline Unknown medical history & $19(5.7)$ & $10(4.2)$ & $9(9.4)$ & 0.072 \\
\hline Other treatment before rt-PA treatment & $9(2.7)$ & $2(0.8)$ & $7(7.3)$ & 0.003 \\
\hline Uncertainty about (anti)coagulation status & $5(1.5)$ & $1(0.4)$ & $4(4.2)$ & 0.025 \\
\hline \multicolumn{5}{|l|}{ Logistic factors } \\
\hline Incorrect triage & $31(9.3)$ & $16(6.7)$ & $15(15.6)$ & 0.020 \\
\hline Technical problems & $30(9.0)$ & $23(9.7)$ & $7(7.3)$ & 0.673 \\
\hline Difficulties with drip or urinary catheter insertion & $27(8.1)$ & $18(7.6)$ & $9(9.4)$ & 0.658 \\
\hline Insufficient personnel & $18(5.4)$ & $15(6.3)$ & $3(3.1)$ & 0.296 \\
\hline CT scan occupied & $16(4.8)$ & $9(3.8)$ & $7(7.3)$ & 0.255 \\
\hline Laboratory results delayed & $12(3.6)$ & $7(2.9)$ & $5(5.2)$ & 0.337 \\
\hline No medication (rt-PA) available & $12(3.6)$ & $9(3.8)$ & $3(3.1)$ & 1.00 \\
\hline Other logistic factors & $7(2.1)$ & $6(2.5)$ & $1(1.0)$ & 0.678 \\
\hline Waiting for consent & $4(1.2)$ & $2(0.8)$ & $2(2.1)$ & 0.326 \\
\hline
\end{tabular}

Values are n (\%), unless otherwise indicated.

${ }^{a}$ Determined by use of the independent sample t test for age, the Mann-Whitney U test for theNIHSS score on admission and SDT, and the $\chi^{2}$ test for categorical variables. ${ }^{b}$ Blood pressure exceeded 185/110 mm Hg on admission. ${ }^{c}$ Difficulties in making the CT scan due to physical restlessness or agitation, language barriers, consultation of a surgeon because of a recent operation, and diagnostic uncertainty.

Factors Independently Associated with a Delayed DNT

Multivariable regression analysis showed the following factors to independently predict a delayed DNT: uncertainty about symptom onset (OR 6.92, 95\% CI 2.12-22.56), uncontrolled blood pressure (OR 9.06, 95\% CI 3.90-21.02), fluctuating neurological deficit (OR 11.33, 95\% CI 4.76-26.95), other treatment before IVT (OR 25.57, 95\% CI 4.79-136.7), uncertainty about (anti-)coagulation status (OR 19.27, 95\% CI 1.78-208.3), other patient-related factors (OR 5.21, 95\% CI 2.08-13.04), and incorrect triage (OR 3.77, 95\% CI 1.52-9.38; table 5).

\section{Discussion}

Our results show that with an optimized IVT protocol, a short median DNT can be accomplished, also in the setting of a secondary care hospital and without the loss of accuracy. The median DNT we found is comparable with previously reported short DNTs achieved in single tertiary referral hospitals [2-4]. To the best of our knowledge, it is the first study that also prospectively documented factors delaying the DNT for each individual patient, allowing the 


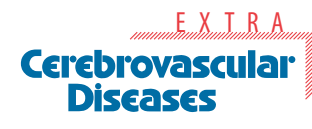

\begin{tabular}{l|l}
\hline \multicolumn{2}{l}{ Cerebrovasc Dis Extra 2015;5:75-83 } \\
\hline DOI: 10.1159/000432405 & $\begin{array}{l}\text { C 2015 S. Karger AG, Basel } \\
\text { www.karger.com/cee }\end{array}$ \\
\hline
\end{tabular}

Van Schaik et al.: Short Door-to-Needle Times in Acute Ischemic Stroke and

Prospective Identification of Its Delaying Factors

Table 5. Factors independently associated with a DNT $>30 \min (\mathrm{n}=334)$

\begin{tabular}{|c|c|c|c|c|}
\hline Variable & $\begin{array}{l}\text { Univariable analysis } \\
\text { OR }(95 \% \mathrm{CI})^{\mathrm{a}}\end{array}$ & $\mathrm{p}$ value ${ }^{\mathrm{b}}$ & $\begin{array}{l}\text { Multivariable analysis } \\
\text { OR }(95 \% \mathrm{CI})^{\mathrm{a}}\end{array}$ & p value ${ }^{b}$ \\
\hline Age, years & $1.01(0.99-1.03)$ & 0.333 & & \\
\hline Males & $1.10(0.68-1.76)$ & 0.701 & & \\
\hline NIHSS score on admission & $0.95(0.91-1.00)$ & 0.045 & $0.99(0.93-1.05)$ & 0.680 \\
\hline SDT, min & $1.00(1.00-1.00)$ & 0.195 & & \\
\hline $\mathrm{SH}$ & $2.82(1.60-4.98)$ & $<0.001$ & $1.96(0.97-3.94)$ & 0.060 \\
\hline \multicolumn{5}{|l|}{ Patient-related factors } \\
\hline Fluctuating neurological deficit & $6.10(2.96-12.54)$ & $<0.001$ & $11.33(4.76-26.95)$ & $<0.001$ \\
\hline Uncontrolled hypertension ${ }^{c}$ & $4.76(2.32-9.77)$ & $<0.001$ & $9.06(3.90-21.02)$ & $<0.001$ \\
\hline Other patient-related factors ${ }^{\mathrm{d}}$ & $2.54(1.17-5.50)$ & 0.018 & $5.21(2.08-13.04)$ & $<0.001$ \\
\hline Uncertainty about symptom onset & $4.71(1.80-12.37)$ & 0.002 & $6.92(2.12-22.56)$ & 0.001 \\
\hline Unknown medical history & $2.36(0.93-6.00)$ & 0.072 & $1.21(0.30-4.89)$ & 0.787 \\
\hline Other treatment before IVT & $9.28(1.90-45.52)$ & 0.006 & $25.57(4.79-136.65)$ & $<0.001$ \\
\hline Uncertainty about (anti)coagulation status & $10.30(1.14-93.42)$ & 0.038 & $19.27(1.78-208.27)$ & 0.015 \\
\hline \multicolumn{5}{|l|}{ Logistic factors } \\
\hline Incorrect triage & $2.57(1.22-5.43)$ & 0.014 & $3.77(1.52-9.38)$ & 0.004 \\
\hline Technical problems & $0.74(0.31-1.78)$ & 0.494 & & \\
\hline Difficulties with drip or urinary catheter insertion & $1.26(0.55-2.92)$ & 0.583 & & \\
\hline Insufficient personnel & $0.48(0.14-1.70)$ & 0.254 & & \\
\hline CT scan occupied & $2.00(0.72-5.54)$ & 0.181 & & \\
\hline Laboratory results delayed & $1.81(0.56-5.86)$ & 0.320 & & \\
\hline No medication (rt-PA) available & $0.82(0.22-3.10)$ & 0.771 & & \\
\hline Other logistic factors & $0.41(0.05-3.43)$ & 0.408 & & \\
\hline Waiting for consent & $2.51(0.35-18.10)$ & 0.361 & & \\
\hline
\end{tabular}

${ }^{\text {a }}$ OR for DNT ( $\leq 30 \mathrm{~min} />30 \mathrm{~min}$ ) and its $95 \%$ Wald CI. ${ }^{\mathrm{b}} \mathrm{p}$ value of the Wald $\chi^{2}$ test. ${ }^{\mathrm{c}}$ Blood pressure exceeded $185 / 110 \mathrm{~mm}$ $\mathrm{Hg}$ on admission. ${ }^{\mathrm{d}}$ Difficulties in making the CT scan due to physical restlessness or agitation, language barriers, consultation of a surgeon because of a recent operation, and diagnostic uncertainty.

identification of independent factors delaying the DNT. Ideally, the recognition of such factors leads to corrective improvement measures. For example, a logistic factor such as incorrect triage in the emergency department could prompt dedicated training programs to improve this. The patient-related factors delaying the DNT seem to be more difficult to tackle but need further appraisal. Uncertainty about symptom onset might be decreased by instructions and training for ambulance personnel and referring general practitioners to verify this more thoroughly before the patient is transported. Indeed, taking the history during patient transport has been helpful $[2,12]$. Uncertainty about (anti-)coagulation status, especially with the introduction of direct oral anticoagulants, is likely to be an increasing problem and stresses the need for a centralized registration accessible for health care workers. Another independent patient-related delaying factor was fluctuating neurological deficit. This is important in the light of the possibly relatively unknown observation that a substantial number of patients with mild or rapidly improving stroke symptoms end up with a poor final clinical outcome, particularly those with persistent large-artery occlusion [13-17]. Together with the evidence that rt-PA treatment is effective irrespective of stroke severity, this should prompt a more aggressive IVT approach [1]. Finally, an uncontrolled blood pressure was independently associated with a delayed DNT. Although many neurologists actively lower the blood pressure to enable rt-PA treatment [18], we anticipate a spontaneous drop as active lowering potentially compromises the already ischemic penumbra [19]. 
Van Schaik et al.: Short Door-to-Needle Times in Acute Ischemic Stroke and Prospective Identification of Its Delaying Factors

Our study has some limitations. First, some data were collected retrospectively and we do not have data on how many stroke patients were not treated with rt-PA due to factors delaying rt-PA treatment and thus not registered. The primary parameters, however, were all prospectively collected, and although missing data on delaying factors in patients not treated with rt-PA could have led to an underestimation of the delaying factors, we have no reason to think that this would change the outcome. Second, we only documented the frequency of a delaying factor and not the net number of minutes lost with every factor delaying rt-PA treatment. Third, the use of the pragmatic symptomatic ICH definition could have led to an underestimation of the complication rate, since it is likely that patients with rapidly progressive stroke symptoms or severe neurological deficit have not always received a follow-up CT scan. Finally, this is an observational study not allowing for conclusions on the causality of the associations. However, we feel it is a good reflection of clinical practice and supports the previously stated view that current DNT targets $[7,8]$ are no longer ambitious enough. In an era with rapidly developing, often elaborate (endovascular) acute stroke therapies, significant clinical gain can still be achieved relatively easily by simply implementing what we already know what is best: to shorten the DNT.

\section{Disclosure Statement}

The authors have no conflicts of interest to disclose.

\section{References}

1 Emberson J, Lees KR, Lyden P, Blackwell L, Albers G, Bluhmki E, et al: Effect of treatment delay, age, and stroke severity on the effects of intravenous thrombolysis with alteplase for acute ischaemic stroke: a meta-analysis of individual patient data from randomised trials. Lancet 2014;384:1929-1935.

-2 Meretoja A, Strbian D, Mustanoja S, Tatlisumak T, Lindsberg PJ, Kaste M: Reducing in-hospital delay to $20 \mathrm{~min}$ in stroke thrombolysis. Neurology 2012;79:306-313.

-3 Meretoja A, Weir L, Ugalde M, Yassi N, Yan B, Hand P, et al: Helsinki model cut stroke thrombolysis delays to 25 min in Melbourne in only 4 months. Neurology 2013;81:1071-1076.

4 Köhrmann M, Schellinger PD, Breuer L, Dohrn M, Kuramatsu JB, Blinzler C, et al: Avoiding in hospital delays and eliminating the three-hour effect in thrombolysis for stroke. Int J Stroke 2011;6:493-497.

5 Fonarow GC, Zhao X, Smith EE, Saver JL, Reeves MJ, Bhatt DL, et al: Door-to-needle times for tissue plasminogen activator administration and clinical outcomes in acute ischemic stroke before and after a quality improvement initiative. JAMA 2014;311:1632-1640.

6 Reeves MJ, Fonarow GC, Smith EE, Pan W, Olson D, Hernandez AF, et al: Representativeness of the Get With The Guidelines-Stroke Registry: comparison of patient and hospital characteristics among Medicare beneficiaries hospitalized with ischemic stroke. Stroke 2012;43:44-49.

7 Jauch EC, Saver JL, Adams HP, Bruno A, Connors JJB, Demaerschalk BM, et al: Guidelines for the early management of patients with acute ischemic stroke: a guideline for healthcare professionals from the American Heart Association/American Stroke Association. Stroke 2013;44:870-947.

$\checkmark 8$ Fonarow GC, Smith EE, Saver JL, Reeves MJ, Hernandez AF, Peterson ED, et al: Improving door-to-needle times in acute ischemic stroke: the design and rationale for the American Heart Association/American Stroke Association's Target: Stroke Initiative. Stroke 2011;42:2983-2989.

-9 Fonarow GC, Smith EE, Saver JL, Reeves MJ, Bhatt DL, Grau-Sepulveda MV, et al: Timeliness of tissue-type plasminogen activator therapy in acute ischemic stroke: patient characteristics, hospital factors, and outcomes associated with door-to-needle times within 60 minutes. Stroke 2011;123:750-758.

10 Mikulík R, Kadlecová P, Czlonkowska A, Kobayashi A, Brozman M, Svigelj V, et al: Factors influencing in-hospital delay in treatment with intravenous thrombolysis. Stroke 2012;43:1578-1583.

11 Kruyt ND, Nederkoorn PJ, Dennis M, Leys D, Ringleb PA, Rudd AG, et al: Door-to-needle time and the proportion of patients receiving intravenous thrombolysis in acute ischemic stroke: uniform interpretation and reporting. Stroke 2013;44:3249-3253.

12 Strbian D, Soinne L, Sairanen T, Häppölä O, Lindsberg PJ, Tatlisumak T, et al: Ultraearly thrombolysis in acute ischemic stroke is associated with better outcome and lower mortality. Stroke 2010;41:712-716. 
13 Smith EE, Abdullah AR, Petkovska I, Rosenthal E, Koroshetz WJ, Schwamm LH: Poor outcomes in patients who do not receive intravenous tissue plasminogen activator because of mild or improving ischemic stroke. Stroke 2005;36:2497-2499.

14 Nedeltchev K, Schwegler B, Haefeli T, Brekenfeld C, Gralla J, Fischer U, et al: Outcome of stroke with mild or rapidly improving symptoms. Stroke 2007;38:2531-2535.

15 Barber PA, Zhang J, Demchuk AM, Hill MD, Buchan AM: Why are stroke patients excluded from TPA therapy? An analysis of patient eligibility. Neurology 2001;56:1015-1020.

16 Rajajee V, Kidwell C, Starkman S, Ovbiagele B, Alger JR, Villablanca P, et al: Early MRI and outcomes of untreated patients with mild or improving ischemic stroke. Neurology 2006;67:980-984.

17 Ozdemir O, Beletsky V, Chan R, Hachinski V: Thrombolysis in patients with marked clinical fluctuations in neurologic status due to cerebral ischemia. Arch Neurol 2008;65:1041-1043.

18 Bauer A, Limburg M, Visser MC: Variation in clinical practice of intravenous thrombolysis in stroke in the Netherlands. Cerebrovasc Dis Extra 2013;3:74-77.

19 Owens WB: Blood pressure control in acute cerebrovascular disease. J Clin Hypertens 2011;13:205-211. 